Musées, Patrimoine et Culture scientifiques et techniques

$150 \mid 2013$

novembre-décembre 2013

\title{
Évolutions des expositions et transformation des rapports entre l'institution et ses publics
}

\section{Serge Chaumier}

\section{OpenEdition \\ Journals}

Édition électronique

URL : http://journals.openedition.org/ocim/1297

DOI : $10.4000 /$ ocim. 1297

ISSN : 2108-646X

Éditeur

OCIM

Édition imprimée

Date de publication : 29 novembre 2013

ISSN : 0994-1908

Référence électronique

Serge Chaumier, «Évolutions des expositions et transformation des rapports entre l'institution et ses publics », La Lettre de l'OCIM [En ligne], 150 | 2013, mis en ligne le 29 novembre 2015, consulté le 30 avril 2019. URL : http://journals.openedition.org/ocim/1297; DOI : 10.4000/ocim.1297

Ce document a été généré automatiquement le 30 avril 2019

Tous droits réservés 


\title{
Évolutions des expositions et transformation des rapports entre l'institution et ses publics
}

\author{
Serge Chaumier
}

1 Les musées changent, les publics se diversifient, et de nouveaux rapports entre les institutions et les usagers se développent. Les pratiques culturelles évoluent et les expositions surtout recouvrent de nouvelles fonctions. De moins en moins orientée vers des motifs éducatifs et pédagogiques, l'exposition s'invente autrement et se déploie avec de nouveaux visages. Loin d'un effet de mode, les nouvelles tendances concrétisent une certaine logique historique. Les connaissances sont désormais disponibles ailleurs, en ligne notamment, et le public vient chercher autre chose dans une exposition. L'émergence des dispositifs immersifs, des expositions fortement scénographiées, des approches interprétatives, des co-constructions... sont autant de signes de ces évolutions. S'inventent ainsi de nouvelles missions pour les institutions et des enjeux que celles-ci doivent affronter. Les processus de conception des expositions, les métiers, les compétences doivent ainsi s'actualiser en conséquence. 


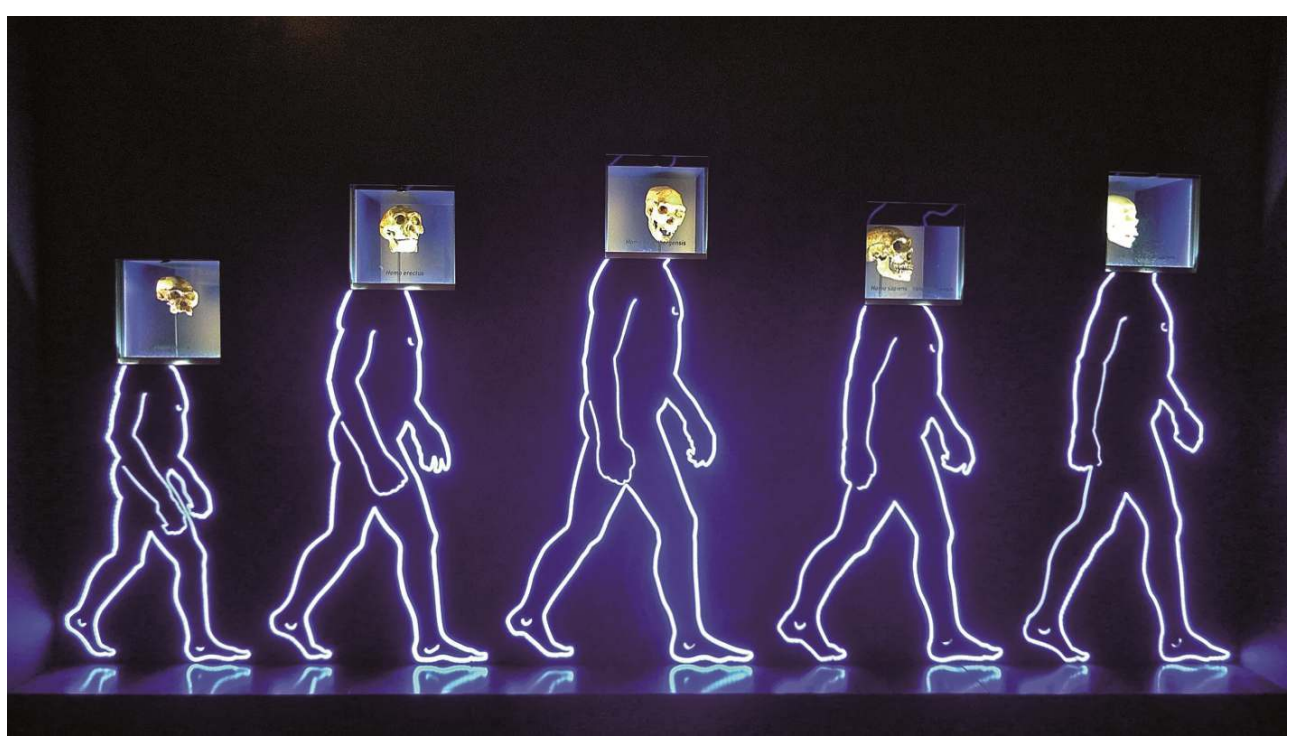

Exposition Le Propre du singe au muséum de Neuchâtel en 2008

(c) S. CHAUMIER

2 Le musée hérite d'une histoire. Il s'inscrit également dans des transformations. En prise avec son époque, il doit s'adapter et pour cela inventer de nouvelles approches, occuper une place différente dans la société. Plusieurs crises ont actuellement des répercussions sur l'institution et conduiront à inventer des formes nouvelles. Crise économique et financière d'abord, crise environnementale, crise politique et sociale, mais aussi crise du sens même de l'action culturelle, qui conduisent de manière conjuguée à devoir innover pour renouveler la place, le rôle et les fonctions du musée ${ }^{1}$. Bien que cela ne soit pas sans lien avec les crises énoncées précédemment, nous voudrions insister ici sur la relation qui nous semble être en train de se développer entre les institutions et leurs publics.

3 Sans écrire ici une histoire bien analysée déjà par ailleurs, on peut rappeler que si le musée est d'abord un projet de partage et de cohésion sociale, d'affirmation du collectif de la Nation réconciliée dans une eschatologie républicaine, il est aussi un lieu de formation des élites, celles-là même qui se doivent de conduire le peuple vers le progrès ${ }^{2}$. Cette mission donnée au musée, incarnée tout particulièrement par le muséum central des arts, le Louvre, est partagée par l'ensemble des autres institutions phares. Le muséum d'Histoire naturelle sera le lieu de formation des savants, le musée des Monuments français inspirera les jeunes architectes et le Conservatoire national des Arts et Métiers, les ingénieurs. Si bien qu'artistes, savants, architectes et ingénieurs disposent de lieux appropriés pour se former, et par conséquent il est naturel que des laboratoires y prennent place, puisque la formation de haut niveau ne peut se penser sans une activité de recherche et de production de connaissances. Lieu de collectes et de conservation, de recherche et de formation des esprits, de fortification d'une conscience éclairée, constituent des fondamentaux historiques. Le musée est le lieu par excellence de l'éducation, dans la mesure où celle-ci est comprise comme un éveil tout au long de la vie à une conscience éclairée, celle qui permet de forger des citoyens responsables.

4 Cette notion d'éducation va voir son sens évoluer avec son appropriation par le ministère chargé jusque-là de l'instruction, c'est-à-dire de l'acquisition des savoirs fondamentaux. Dans les années d'après-guerre, les musées, sous l'égide de l'UNESCO, s'ouvrent à un plus large public, et s'orientent massivement à recevoir des publics scolaires en place des étudiants ${ }^{3}$. En déployant une visée éducative, confondue bien souvent avec une mission 
d'instruction, le musée envisage ses objectifs dans la transmission de contenus informatifs. À tel point que le souci formulé durant longtemps par les conservateurs s'enquérant de mieux connaître les publics sera de se demander ce qu'ils ont appris. Les évaluations des expositions devront montrer qu'il se joue bien d'autres choses dans les espaces. Les années cinquante à soixante-dix verront se développer les services pédagogiques, l'accueil des scolaires et le détachement d'enseignants susceptibles de mettre en adéquation les offres du musée au regard des programmes.

5 Les visiteurs ont si bien intégré que le musée était un lieu dévolu aux savoirs légitimes, où sévissaient des experts qui déroulaient des connaissances à partir de collections, que la plupart des études conduites auprès des publics ont pu faire apparaître et continuent bien souvent à relater cette motivation proclamée de "vouloir apprendre ». Ce motif louable s'il en est représente néanmoins une approche particulière qui n'est pas nécessairement communément partagée et qui semble même l'être de moins en moins. Il faut pour le comprendre replacer cette motivation dans une histoire longue des politiques culturelles et de la signification donnée au terme de culture ${ }^{4}$. Si l'éducation populaire avait des ambitions bien plus larges que de seulement démocratiser l'accès à des savoirs, il n'empêche qu'une relation étroite est longtemps maintenue entre l'instruction et l'éducation. Si André Malraux cherche à faire comprendre la place de chacune des institutions, l'école ou l'université d'une part, le musée ou le théâtre de l'autre, des confusions s'instaurent. "Apprendre et apprendre à aimer", pour le résumer simplement, or les institutions culturelles tendent bien souvent à oublier l'amour.

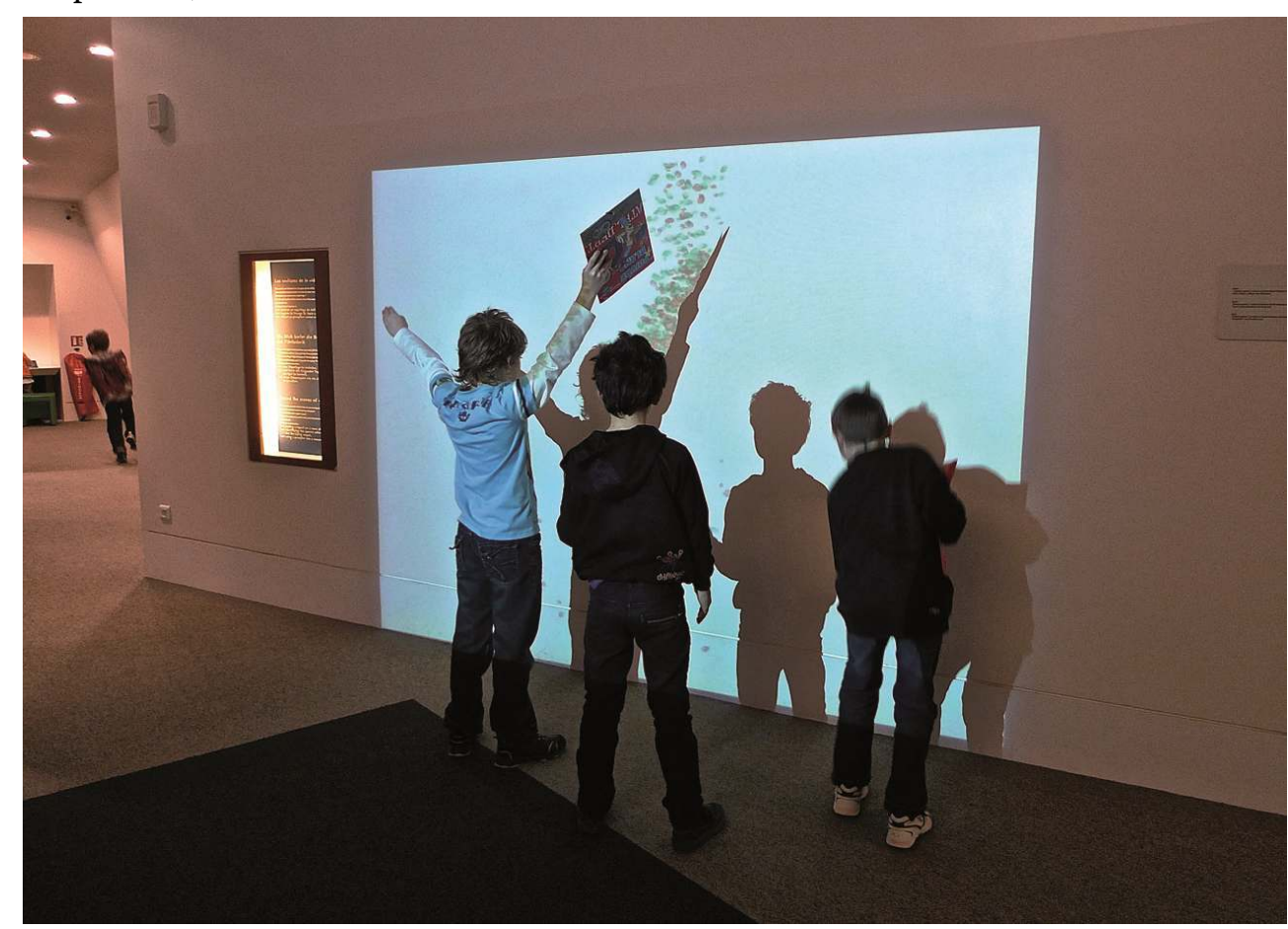

Le Vaisseau à Strasbourg, exposition permanente

(C) S. CHAUMIER

Prenant conscience que les publics doivent être accompagnés, que leur bagage culturel d'origine ne suffit pas toujours, et de moins en moins avec l'éloignement de l'école de la culture classique, à appréhender les contenus proposés, les musées vont investir dans les outils de médiation, pour expliquer, développer, agrémenter les savoirs afin de les rendre plus digestes et plus adaptés aux publics envisagés. Cet effort louable, qui va prendre des 
formes diverses, tend à rendre plus opérationnelle l'acquisition des connaissances. Même si l'action culturelle apportera d'autres visions et si la nouvelle muséologie élargira le spectre de ce qui est colporté, intégrant par exemple des approches sensitives afin de ne pas résumer l'apprentissage aux contenus intellectuels, il demeure que massivement les outils de médiation sont pensés comme des supports d'un discours informatif. Les musées sont entrés dans l'ère de la communication, comme le résume Jean Davallon ${ }^{5}$. Depuis trente ans se développent ainsi de nouvelles formes, plus interactives par exemple, mais portées à développer une approche assez similaire de transmission.

7 Pensons aux fiches pédagogiques en salle, aux cartels développés, aux multimédias complétant la galerie d'étude du musée national des Arts et Traditions populaires, aux films didactiques, aux panneaux de textes de plus en plus affinés pour être compréhensibles d'un plus large public, aux dispositifs sonores sous forme d'audioguides, aux visites guidées bien évidemment, et même aux interactifs et aux manips, familiers des musées de science ou d'archéologie, ces propositions de plus en plus nombreuses, diversifiées, inventives et de plus en plus techniques, nécessitant des compétences pointues, sont devenues évidentes et même banales. Ce que l'on regroupe sous le terme générique et fourre-tout de médiation est devenu le lot commun de toutes les expositions. Il faut revoir les images anciennes des salles de musée d'il y a encore 50 ans pour mesurer tout le chemin parcouru. Pourtant la majeure partie de ces propositions réitèrent la même mission, prétendre informer et communiquer des contenus visant à l'acculturation des utilisateurs.

8 Pourtant ces démarches ne résument pas tout. Elles ne peuvent occulter que les institutions ont développé bien d'autres démarches qui ne visaient pas directement à l'apprentissage, mais dont celui-ci n'était que la résultante. Car c'est ici que s'opère la différenciation, lorsque l'objectif est directement de transmettre un contenu ou quand l'apprentissage est la conséquence d'autres choses. Car on apprend de bien des manières et avec bien des différences, danser c'est apprendre un pas de danse ou une technique du corps, chanter c'est savoir poser sa voix, mais il n'échappera pas que la motivation n'est pas d'abord celle-ci quand il s'agit de prendre du plaisir. Ainsi les ateliers de pratique artistique, les cafés des sciences où l'on débat, les visites nocturnes des salles d'exposition, les jeux parcours découverte, les rencontres et les visites contées, l'émergence du spectacle vivant dans les salles, le montage d'une fusée avec un groupe d'adolescents dans un musée de science... Bien des occasions, innombrables, sont offertes d'apprendre par effets induits, mais dont la raison première est de se retrouver, de nouer des relations, de débattre, de se rencontrer, d'inventer ensemble, de créer... 


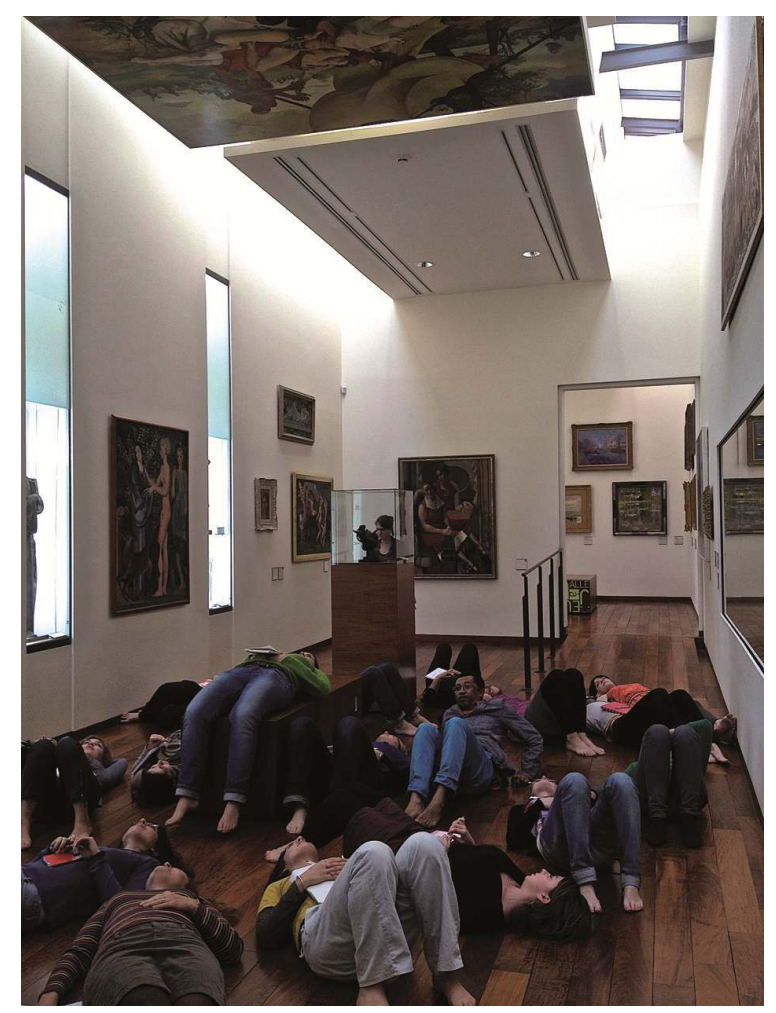

Atelier « Parcours des sens » mené avec les étudiants du master expographie de l'université d'Artois au musée de La Piscine à Roubaix en 2012.

(c) S. CHAUMIER

9 Car toutes les études de public rappellent que les motivations des visiteurs sont en réalité plurielles, hétérogènes et loin des stéréotypes. Selon les occasions, selon les lieux, les moments, les envies, chez une même personne s'exprimera le désir d'apprendre peutêtre, mais aussi et surtout de découvrir, de se questionner, d'être intrigué, provoqué, de prendre du plaisir, de partager, d'échanger, de jouer, de s'amuser... Bref, la visite au musée est loin d'être une visite studieuse, un complément à la formation scolaire, mais un moment unique d'émotion, de contemplation, de divertissement, d'amusement. Et cela n'est pas antinomique avec le fait que l'on apprenne au passage quelque chose. Ce qui est peut-être nouveau, c'est que ce ne soit plus la raison suffisante, et peut-être même pas la raison première. Bien sûr cela dépend des lieux, des expositions, des situations, mais il apparaît que les motivations sont plus diverses et moins centrées sur l'informatif. Ceci est du reste assez logique.

L'information est désormais présente partout. Omniprésente même. Chacun peut l'avoir à portée de clic, sur son smartphone au cœur même des salles d'exposition, davantage encore en restant chez lui en consultant son ordinateur, et pas seulement les encyclopédies d'antan. Dès lors, pourquoi venir dans les institutions culturelles? Pas plus que la visite à la bibliothèque ne peut désormais se justifier par la seule consultation du livre, alors que c'est une occasion d'échanges et de partages, la visite au musée doit être d'un rapport radicalement différent à celui que l'on imaginait jusque-là ${ }^{6}$. Dans ce sens, le musée n'est plus destiné à être un lieu de transmission d'informations, mais un vecteur de mise en questionnement et en organisation particulière de ces informations. C'est-àdire que chaque exposition doit lui donner un sens, une approche singulière et unique. C'est pour cette raison que l'exposition temporaire s'est développée depuis 30 ans et a pris le pas sur les espaces permanents, car on y vit un événement. Chaque fois différente, 
la visite est chargée de surprises. L'exposition ne peut plus être un simple outil de mise en communication, car c'est là une approche trop restrictive, elle doit être une mise en dialogue et en débat, mais aussi en contexte et en expériences. Le musée devient dialogique ${ }^{7}$.

On a dit parfois que pour retrouver un contact avec la matérialité, le musée pourrait se voir réinvesti de sens dans une époque où le virtuel devient prédominant, mais cela n'est pas suffisant. Si l'on peut apprécier le rapport à l'objet authentique, l'exposition ne peut plus être ou redevenir un lieu de simple monstration. Elle ne peut plus se contenter de montrer, elle doit démontrer, accompagner et construire un rapport inattendu. C'est en ce sens que l'on peut comprendre la montée en puissance de la scénographie d'exposition depuis trente ans. De plus en plus les expositions offrent des occasions uniques et singulières. L'expérience du visiteur devient le maître mot pour lui faire vivre un moment exceptionnel. Ceci suppose des moyens et des compétences de plus en plus techniques. Ainsi la scénographie n'est pas un luxe ou une mode, elle devient le fondement même de l'expérience engendrée chez le visiteur. Celui-ci vit l'exposition moins pour son rapport à un objet, une collection, et moins encore pour un approfondissement de son savoir, mais pour une confrontation avec un ensemble qui va engendrer le rapport à l'objet et au savoir. Non comme motif premier, mais comme résultante.

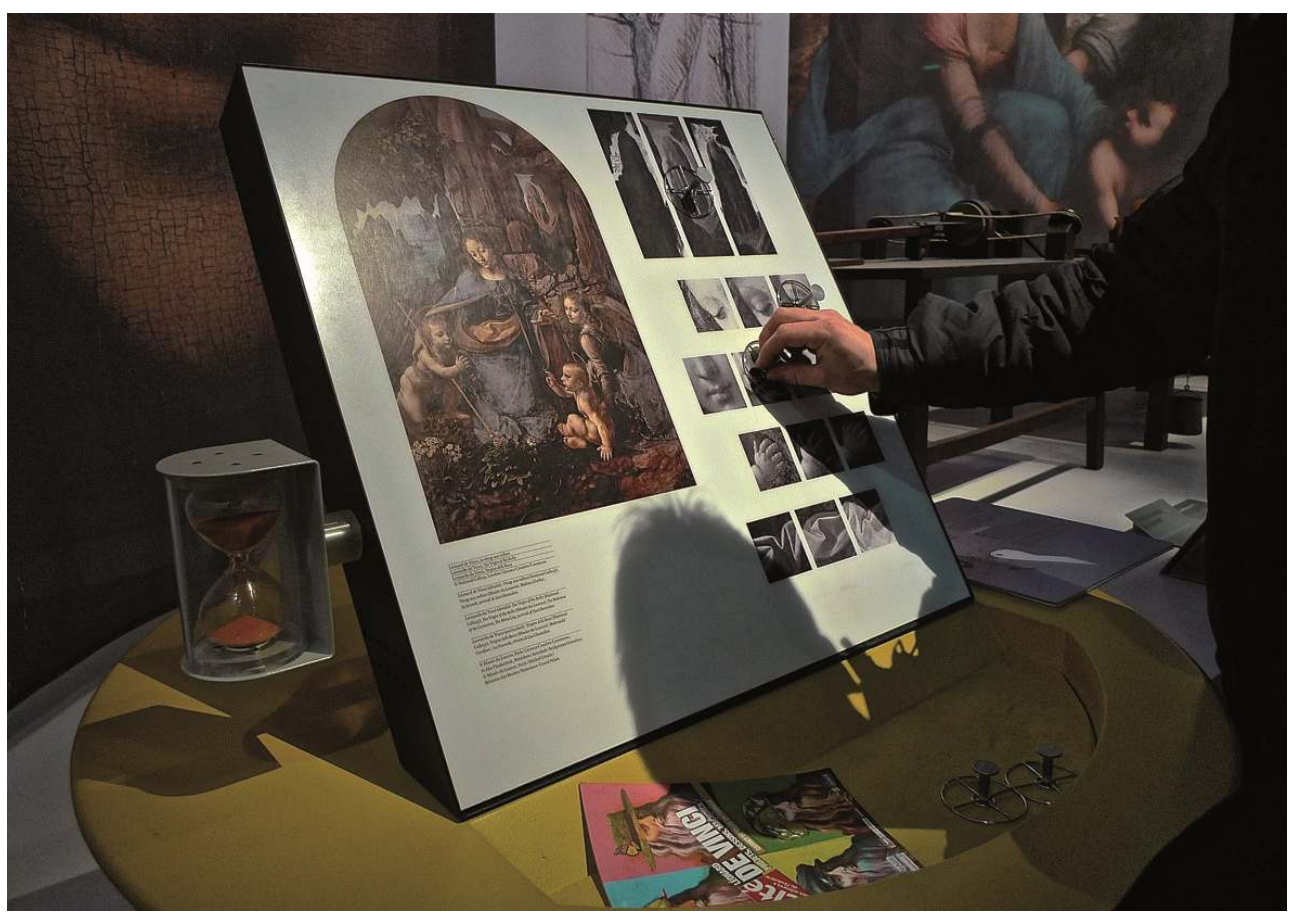

Présentation interactive dans l'exposition Léonard de Vinci, projets, dessins, machines proposée en 2013 par la Cité des Sciences et de l'Industrie, Universcience.

(C) S. CHAUMIER

12 Ainsi visite-t-on de plus en plus les expositions pour elles-mêmes, davantage que pour ce qui les compose. Ceci s'opère à deux niveaux ${ }^{8}$. En premier lieu par ce qu'elles disent, le discours tenu, ce que l'on doit nommer le programme muséographique. Si la thèse est originale, le propos impertinent ou étonnant, l'approche singulière, alors l'exposition fait mouche. Les expositions de Jean Clair en sont les archétypes. Le visiteur ne se presse pas pour voir tel ou tel objet dans l'exposition La Mélancolie, mais pour se confronter au propos d'un auteur. En second lieu, l'exposition prend sa force par son ensemble, ce 
qu'elle dégage de cohérence entre le fond et la forme choisie, en l'occurrence son projet scénographique. Ces deux éléments lorsqu'ils sont savamment composés l'emportent. Ce n'est plus la collection qui suffit à faire sens, pas même l'information et les savoirs dispensés, mais la manière dont l'ensemble est relié et proposé. L'exposition prend alors son autonomie et devient œuvre en soi, comme en atteste l'histoire de l'exposition depuis Harald Szeemann à la Biennale de Berne en 1969, mais également le développement du droit de la propriété intellectuelle dans les productions d'exposition.

Qui n'a pas visité une exposition pour l'expérience qu'elle procure davantage que pour ce qu'elle entend promouvoir? Qui ne s'est pas surpris à manipuler un nouvel outil de médiation, telle une table tactile, d'abord pour expérimenter la manière de s'en servir et l'interface, avant que de se pencher éventuellement sur les informations dispensées? L'apprentissage des supports techniques n'est pas le moindre des apprentissages que réalise le visiteur, et l'on sait par exemple que les personnes âgées trouvent dans les musées une socialisation efficace aux nouvelles technologies. Ainsi, que ce soit par le dispositif en particulier, ou par l'ambiance générale dégagée par l'exposition, l'expérience du visiteur prime et participe de son plaisir. Ce que l'on a nommé les expositions d'immersion signifie bien cette suprématie de l'exposition sur ce qui la compose. Si cela peut sembler une évidence, le visiteur est plus que jamais un visiteur de l'exposition, avant que d'être un visiteur des éléments qui participent de l'exposition, collections ou savoirs.

14 S'il appartient à l'institution de se porter garante des contenus, de signifier une lecture adéquate des collections et des connaissances dont on dispose, cela relativise malgré tout énormément les enjeux. C'est peut-être moins dès lors la prétendue authenticité de l'objet qui importe, le détail de la précision historique ou scientifique, que l'expérience que l'on propose de vivre au visiteur. En devenant vecteur d'expériences, et même opérateur des propositions expérientielles, les concepteurs de l'exposition se transforment de scientifiques en animateurs, davantage du reste qu'en médiateurs, ce concept se référant davantage dans le langage courant à des transmetteurs d'informations. Il s'agit moins de résoudre des conflits potentiels que de donner à vivre, d'insuffler une âme. Pour cela, trois options semblent se dégager :

- Une des premières approches vise à penser un produit exposition qui place le visiteur en récepteur d'une proposition pensée pour lui, et qui lui fournira une expérience atypique. Celle-ci peut bien être interactive, il n'empêche qu'il y a une frontière étanche et dichotomique entre l'équipe de conception et les destinataires. Elle engage à un dialogue entre le fond et la forme, qui dans les cas réussis dégage un sens nouveau. L'exposition peut être formidablement scénographiée et engager un rapport de fascination par son emprise et sa spectacularisation. C'est à l'excès le modèle du show dans les expositions universelles. La relation entre l'architecture et la scénographie est alors majeure et peut confiner au produit de consommation. Elle s'adresse avant tout à des publics. 


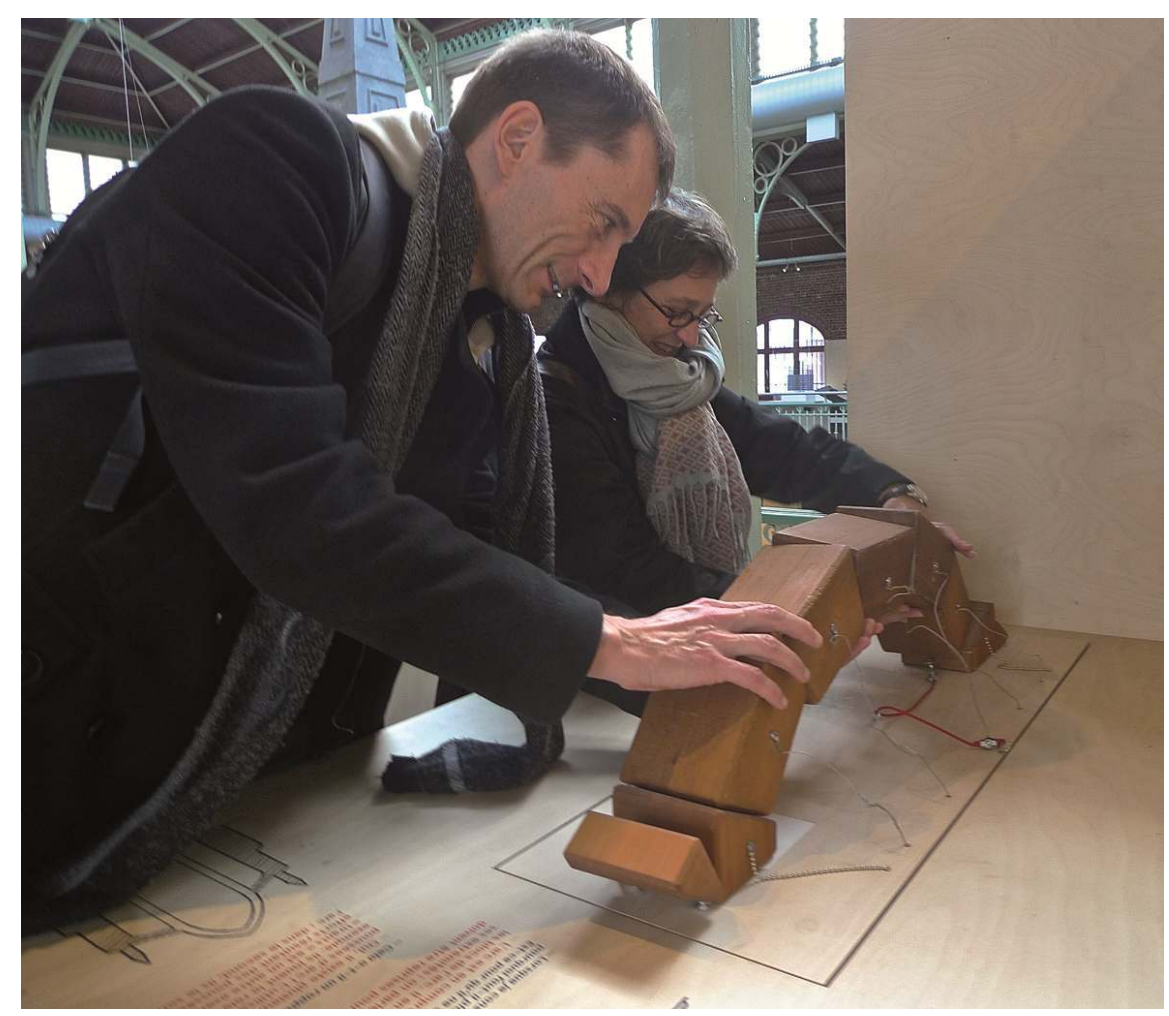

Comment tout cela tient ? Exposition d'architecture (commissaire Michel Provost) aux Halles SaintGery à Bruxelles en 2012

(C) S. CHAUMIER

- La seconde approche est radicalement différente dans ses attendus Elle repose sur des principes et une histoire de l'action culturelle qui conduit à placer le visiteur en situation d'acteur. En proposant de s'investir, c'est d'abord la mise en relation qui est envisagée, les retombées en terme d'apprentissage ne sont qu'espérées comme conséquences induites. Ainsi, les collections et les savoirs ne sont que des opportunités et des prétextes pour construire des liens dialectiques entre des femmes et des hommes composant la société et leur donner ainsi une occasion de mieux vivre ensemble, en s'appartenant davantage. C'est le fondement même de toute action culturelle qui se développe souvent à partir ou en marge de l'exposition. Elle met en action des usagers.

- La troisième approche peut être comprise comme un développement de la précédente. Particulièrement actuelle, elle envisage la co-construction des actions et la participation à la fois comme un moyen et un objectif. Sur le modèle du Wiki, il s'agit de construire des collectifs producteurs de formes et de contenus. En cohérence avec l'évolution du rapport au savoir et à l'expertise, elle engage une démocratisation généralisée. Encore balbutiante dans le domaine de l'expologie, des tendances s'affirment qui paraissent en cohérence avec l'histoire et le développement actuel des institutions'. Elles sont l'occasion de réconcilier des pôles jusque-là distendus, par exemple la production des savoirs et leur médiation, dans une visée systémique. La production même des expositions peut alors s'envisager selon des principes collaboratifs. Elle implique des contributeurs.

Ces trois modalités sont des sources de renouvellement des institutions. Dans les trois cas, il paraît que les aspects patrimoniaux, à savoir les collections et les savoirs scientifiques, sont déterminés par des motifs transcendants, à savoir l'expérience vécue, les relations engagées, les co-constructions élaborées. Le patrimoine ne suffit pas à faire sens, il doit être au service d'autres choses. Cela ne signifie pas que la nécessité de la collection et de 
la conservation ou de la production des savoirs disparaisse, mais qu'elle est actualisée au profit de ce que le musée ne devrait jamais perdre de vue, le bénéfice de tous.

Ces évolutions muséales, et plus particulièrement de la place dédiée aux usagers, se trouve avoir des répercussions non seulement sur le sens dévolu aux institutions, ainsi que sur les dimensions épistémologiques de production des connaissances, du rapport du citoyen avec l'expertise, de la délégation à autrui de la fabrication et de la transmission des biens culturels, de la légitimité de parole, mais aussi de la place et du rôle des professionnels eux-mêmes. Il convient de repenser les compétences nécessaires, et par conséquent les formations qui envisagent les métiers de demain. Ce n'est pas un hasard si se développent par exemple les métiers de webmaster ou de community manager dans les institutions, c'est un rôle qui sera de plus en plus prédominant avec les digitals natives. Mais c'est aussi un rééquilibrage alors que le médiateur se métamorphose en animateur de mises en relation et propulseur d'actions, activateur de convergences, distillateur de créativités, interprétateur de subjectivités ${ }^{10} \ldots$ L'externalisation des fonctions auprès de professionnels spécialisés dans les processus de production des expositions ${ }^{11}$ rend également compte de ce mouvement qui va de la conservation - recherche à la production - à l'activation des compétences de tout un chacun. De même que l'enseignement devrait se réformer pour s'adapter à ces nouvelles tendances, le musée est en train de vivre sa mue.

\section{NOTES}

1. Chaumier, S. et Porcedda, A. (dir.) Musées et développement durable. La Documentation française, 2011.

2. Poulot, D. Une histoire des musées de France, XVIII-XX ${ }^{\mathrm{e}}$ siècle. La Découverte, 2008. Mairesse, F. Le Musée, temple spectaculaire. PUL, 2002.

3. Chaumier, S. «Éducation » in Desvallées, A. et Mairesse, F. (dir.) Dictionnaire encyclopédique de muséologie. Armand Colin, 2011.

4. Chaumier, S. L'inculture pour tous. La Nouvelle utopie des politiques culturelles. L'Harmattan, 2010.

5. Davallon, J. L'exposition à l'œuvre. Stratégies de communication et médiation symbolique. L'Harmattan, 1999.

6. Couzinet V. (dir.) Bibliothèques et musées : notions et concepts communs, Culture et Musées, $\mathrm{n}$ - 21, Actes Sud, 2013.

7. Meunier, A. (dir.) La muséologie, champ de théories et de pratiques. Presses de l'université du Québec, 2012.

8. Pour de plus larges développements voir : Traité d'Expologie. Les Écritures de l'exposition. La Documentation française, 2012.

9. Chevalier, D. et Fanlo, A. Métamorphoses des musées de société. La Documentation française, 2013.

10. Chaumier, S. et Mairesse, F. La Médiation culturelle. Armand Colin, 2013.

11. Manuel de conception des expositions. Téléchargeable sur le site de l'Association des scénographes, www.scenographes.fr 


\section{RÉSUMÉS}

Aujourd'hui la conception des expositions a changé et désormais les logiques immersives, expérientielles ont succédé aux volontés d'expérimentation, d'interactivité, tandis que les approches participatives et collaboratives se répandent: l'auteur dresse le constat de ces évolutions, puis montre en quoi s'inventent ainsi de nouvelles missions pour les institutions et les enjeux que celles-ci doivent affronter pour relever ces défis.

INDEX

Keywords : Expositions

\section{AUTEUR}

\section{SERGE CHAUMIER}

Serge Chaumier est professeur des universités, responsable du master Expographie Muséographie à l'université d'Artois. serge.chaumier@univ-artois.fr 